\title{
Meyer's Loop Anatomy Demonstrated Using Diffusion Tensor MR Imaging and Fiber Tractography at 3T
}

\author{
Goga Cristina1,2,3, Firat Zeynep², Brinzaniuc Klara1, Florian IS³ \\ 1 University of Medicine and Pharmacy Tirgu Mures, Tirgu Mures, Romania, Department of Anatomy \\ 2 Yeditepe University School of Medicine, Istanbul, Turkey, Department of Neurosurgery \\ ${ }^{3}$ Cluj County Emergency Hospital, Cluj Napoca, Romania, Department of Neurosurgery
}

\begin{abstract}
Objective: The ultimate anatomy of the Meyer's loop continues to elude us. Diffusion tensor imaging (DTI) and diffusion tensor tractography (DTT) may be able to demonstrate, in vivo, the anatomy of the complex network of white matter fibers surrounding the Meyer's loop and the optic radiations. This study aims at exploring the anatomy of the Meyer's loop by using DTI and fiber tractography.

Methods: Ten healthy subjects underwent magnetic resonance imaging (MRI) with DTI at $3 \mathrm{~T}$. Using a region-of-interest (ROI) based diffusion tensor imaging and fiber tracking software (Release 2.6. Achieva, Philips), sequential ROI were placed to reconstruct visual fibers and neighboring projection fibers involved in the formation of Meyer's loop. The 3-dimensional (3D) reconstructed fibers were visualized by superimposition on 3-planar MRI brain images to enhance their precise anatomical localization and relationship with other anatomical structures. Results: Several projection fiber including the optic radiation, occipitopontine/parietopontine fibers and posterior thalamic peduncle participated in the formation of Meyer's loop. Two patterns of angulation of the Meyer's loop were found.
\end{abstract}

Conclusions: DTI with DTT provides a complimentary, in vivo, method to study the details of the anatomy of the Meyer's loop.

Keywords: DTI tractography, Meyer's loop, optic radiation, projection fibers, white matter anatomyIntroduction

Received: 05 August 2014 / Accepted: 29 October 2014

Meyer's loop is commonly known as the temporal loop of the anterior fibers of the optic radiation that originate in the lateral geniculate body and course anteriorly, into the temporal lobe, before turning posteriorly to reach the occipital visual cortex. Adolf Meyer applied histological, brain lesion studies to describe this peculiar course into the temporal lobe of the visual projection fibers, in 1907 [1]. Histological staining techniques that are applicable in humans provide an ambiguous visualization of the myelinstained white matter fibers and are limited by an inability to precisely trace and reconstruct the course of these fibers. Therefore, the composition and course of the Meyer's loop, or the fact that this temporal loop is composed exclusively by the optic radiation fibers remains unclear.

A unique $3 \mathrm{D}$ perspective on the white matter architecture can be achieved by applying the fiber dissection technique, but with this technique it is difficult to differentiate individual visual projection fibers, because of the intrinsic limitations of this technique to distinguish and trace individual fibers among the complex network of many neighboring fibers $[2,3]$. Furthermore, the delineation of the Meyer's loop using the fiber dissection technique is challenging and its exact fiber composition and location are a point of controversy. Knowledge of the anatomy of the Meyer's loop is essential for minimizing the risk of damaging this structure during surgery for temporal lobe lesions and epilepsy. However, the ultimate anatomical details of the Meyer's loop remain even today obscure, because both

Correspondence to: Klara Brinzaniuc

E-mail: klara_branzaniuc@yahoo.com histological and fiber dissection techniques are less suitable to accurately demonstrate its precise fiber composition and exact location.

DTI is a MRI technique that can be used to evaluate the directionality of the diffusion of water molecules as they move between the white matter fibers [4-6]. From this information, the main direction and the extent of water diffusion can be determined, and the orientation of white matter fibers indirectly evaluated. This technique, developed more than a decade ago, provides a unique noninvasive, in vivo, visualization of the white mater architecture and offers the basis from which fractional anisotropy (FA) maps and diffusion tensor tractography (DTT) images are generated [7]. The color FA maps depict directionality information of white matter fibers in a 2D image, while DTT has the advantage of displaying a 3D reconstruction of a whole-length fiber tract. The DTT is generated using mathematical algorithms that connect image voxels based on their anisotropy and principal diffusion direction $[6$, 8 -10]. Because DTT offers the only currently available method for a noninvasive investigation of the white matter fibers, it might provide additional data regarding the anatomy of the Meyer's loop [11-16].

Meyer's loop presents several white matter fiber systems in its vicinity, such as the uncinate fasciculus, the occipitofrontal fasciculus, the anterior commissure and the inferior longitudinal fasciculus. In the anterior temporal region all these various fibers intercross and merge with the Meyer's loop and cannot be precisely distinguished in fiber dissection studies. Moreover, optic radiation fibers that allegedly form the Meyer's loop exclusively, have complex relations 
with neighboring projection fibers for the temporal and occipital regions, including the temporopontine fibers, occipitopontine fibers and the posterior thalamic peduncle, and this complicates their differentiation in both histological and fiber dissection studies [1-3]. Few studies of DTT specifically describe these complex relations between the projection fibers that might be involved in the formation of the Meyer's loop such as the temporopontine and occipitopontine fibers, and the posterior thalamic peduncle $[17,18]$. To characterize the course and composition of the Meyer's loop and its relations with related fibers, we studied 10 healthy subjects.

\section{Methods}

Ten healthy subjects, without history of neurological disease, head injury, or psychiatric disorder ( 4 females and 6 males, mean age 36 years, range $16-59$ years) were recruited after obtaining institutional board review approval. We performed MRI using a 3.0-T MR unit (Philips, Achieva, Eindhoven, Netherlands) with an 8-channel sensitivity encoding (SENSE) head coil. DTI was acquired using a single-shot spin echo planar imaging (EPI; TR 10,000 ms, TE $53 \mathrm{~ms}$, EPI factor 67, slice thickness $2.5 \mathrm{~mm}$, gap $0 \mathrm{~mm}$ ). Thirty-two diffusion directions at $b=1000$ seconds $/ \mathrm{mm} 2$ were acquired in addition to $b=0$ images. Sixty slices were taken for whole brain coverage from the vertex to the foramen magnum. The average acquisition time was 6 minutes. The reconstructed voxel size was $0.94 \mathrm{X} 0.94 \mathrm{X} 2.50$ $\mathrm{mm}$. Whole brain T1-weighted 3D turbo field echo (3D T1-TFE), with the same resolution, was acquired in the sagittal plane (TR $8.5 \mathrm{~ms}$, TE $4 \mathrm{~ms}$, slice thickness $1 \mathrm{~mm}$ ) and coregistered for anatomical guidance. T2-weighted turbo spin echo, SENSE encoding (T2-TSE SENSE), images were also acquired in axial plane (TR $80 \mathrm{~ms}$, TE 3000 $\mathrm{ms}$, slice thickness $4 \mathrm{~mm}$ ) to facilitate anatomical guidance.

DTI computed FA maps were individually coregistered with T1-weighted images using the MRI system's workstation (Release 2.6, Achieva, Philips). FA maps were displayed as color-orientation maps (Figure 1). The colorcoding of the white matter fiber tracts in the FA maps was as follows: red for left-right oriented fibers, blue for superior-inferior oriented fibers and green for anterior-posterior oriented fibers.

The fiber tracking software was used to generate 3D DTT. The 3D tract reconstruction was performed using the FACT (Fiber Assignment by Continuous Tracking) method, which is a deterministic method that initiates fiber trajectories from user-defined voxels or seeds $[6,10]$. Anisotropy threshold values for FA and deflection parameters were set between $0.27-0.35$ and $700-915$ (where 0 refers to full deflection and 1000 to no deflection) respectively to obtain the maximum tract conspicuity. A knowledge-based multiple-ROI technique was used $[8,19,20]$. To delineate the relevant white matter fibers, multiple ROIs were placed in the white matter of the occipital region, and the regions of the lateral geniculate body and the optic tract according to previously described anatomical reference methods to identify the lateral geniculate body and optic tracts in T1weighted images and color-coded FA images [21-23]. The colors of the reconstructed tracts on DTT were selected to permit optimal visualization. The 3D reconstructed fibers were visualized by superimposition on 3-planar T1-weighted $3 \mathrm{D}$ turbo field echo (3D T1-TFE) images to enhance their precise anatomical localization and relationship with other anatomical structures.

Six white matter tracts relevant to the Meyer's loop were reconstructed: the posterior thalamic peduncle that includes the optic radiation, the occipitopontine/ parietopontine fibers, the anterior commissure, the occipitofrontal fasciculus, the uncinate fasciculus and the inferior longitudinal fasciculus. To ensure a comprehensive reconstruction off all possible Meyer's loop fibers, a large ROI in the occipital lobe, defined on a coronal image, and a large ROI in the region of lateral geniculate body, including the pulvinar and crus cerebri of the midbrain, and defined on a sagittal image were used (Figure 2). Then, an exhaustive search was performed to identify all the fibers that penetrate both ROIs and participate in the formation of Meyer's loop, and then parcelate these fibers according to their different connections. By using large ROIs instead of directly defining ROIs in the lateral geniculate body and the calcarine visual cortex that were assumed by classical DTI studies to exclusively reconstruct visual projection fibers $[11,21,24-26]$, we hypothesized that our technique should be more accurate in estimating the composition of the Meyer's loop because of the following considerations. First, both histological and fiber dissection studies have shown that the lateral geniculate body was

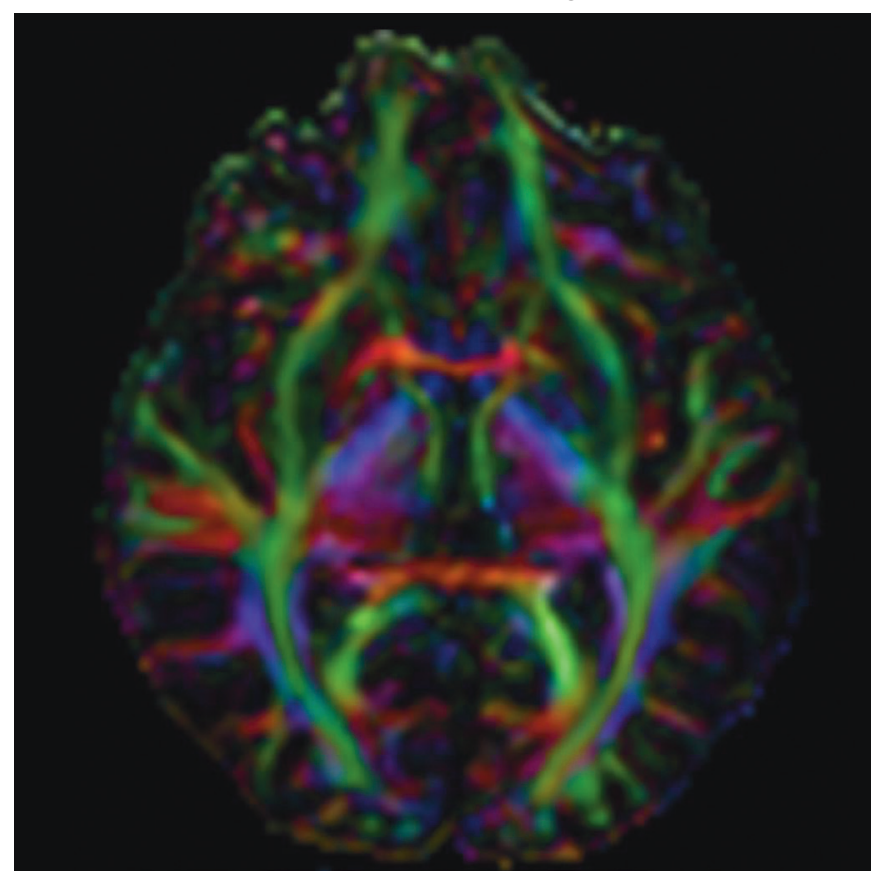

Fig. 1. Fractional anisotropy (FA) maps displayed as color-orientation maps. The color-coding of the white matter fiber tracts in the FA maps is: red for left-right oriented fibers, blue for superior-inferior oriented fibers and green for anterior-posterior oriented fibers. 


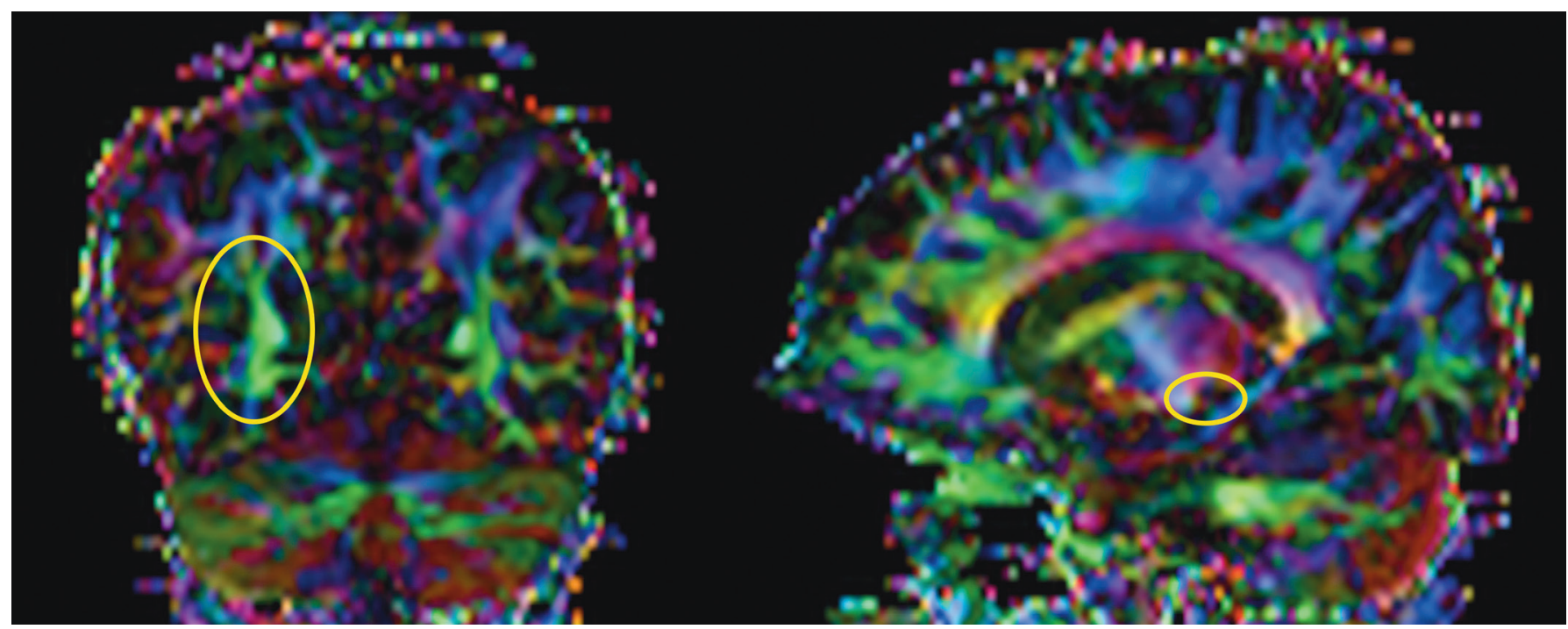

Fig. 2. ROI placement on FA color-coded maps to ensure a comprehensive reconstruction of all possible Meyer's loop fibers. A large ROI in the occipital lobe, defined on a coronal image (left), and a large ROI in the region of lateral geniculate body, including the pulvinar and crus cerebri of the midbrain, and defined on a sagittal image (right).

surrounded by a dense and intricate network of projection fibers among which the visual projection fibers are only one of the various components, so seeding in the region of the lateral geniculate body, including the pulvinar and crus cerebri, has the possibility of including these neighboring projection fibers and we could check whether these projection fibers pass through the Meyer's loop, to ensure that all components of the Meyer's loop are reconstructed and not exclusively the optic radiation fibers [1-3]. Second, we could verify whether other occipital cortical or thalamic projection fibers for the occipital lobes participate in the formation of the Meyer's loop, by delineating a large occipital ROI rather than directly placing a ROI in the calcarine visual cortex.

After Meyer's loop was identified, a ROI in the frontal lobe, using a coronal image, was placed, in addition to the occipital ROI, to reconstruct the occipitofrontal fasciculus. For reconstructing the fibers of the anterior commissure two ROIs were placed $10 \mathrm{~mm}$ apart on the left and right side of the midsagittal plane. The uncinate fasciculus was reconstructed by placing one $\mathrm{ROI}$ in the in the anterior temporal lobe and a second ROI in the white matter of the basal portion of the frontal lobe in the coronal plane.

To verify the reproducibility across observers we involved 2 clinicians as raters (1 radiologist and 1 neurosurgeon). Both of them were shown how to use the software and were asked to independently reconstruct the Meyer's loop and neighboring fibers using our method. The anatomical characteristics of the Meyer's loop and the relations with neighboring fibers were analyzed.

\section{Results}

\section{Course and fiber composition of the Meyer's loop}

Meyer's loop was reconstructed in all subjects. Fibers originating in the region of the lateral geniculate body, including pulvinar and the lateral aspect of crus cerebri of the midbrain, extended in an anterolateral direction into the anterior temporal region, before these fibers looped posteriorly and reached the occipital cortical region (Figure 3). This temporal loop is commonly known as the Meyer's loop. All the fibers composing the Meyer's loop continued their course in the roof of the temporal horn, atrium and occipital horn of the lateral ventricle. In the occipital region, these fibers turned medially, posterior to the occipital horn of the lateral ventricle and reached their cortical terminations.

Several projection fibers participated in the formation of the Meyer's loop. Optic radiation fibers originating in the lateral geniculate body and in continuation with the optic tract passed in the Meyer's loop and continued their course towards the occipital visual cortex. The posterior thalamic peduncle includes thalamocortical projection fibers that originate in the pulvinar and connect the pulvinar of the thalamus with occipital cortical regions. The optic radiation is one of the various projection fibers in the posterior thalamic peduncle. The accurate differentiation between these various posterior thalamic peduncle fibers was extremely difficult, because all these fibers originating either in the lateral geniculate body or in the pulvinar merged and intermingled before forming the Meyer's loop, and could not be distinguished or separated at this level. The occipitopontine fibers, projection fibers originating in the occipital cortex and terminating in the pontine nuclei, coursed in the Meyer's loop together with the optic radiation fibers and thalamocortical projection fibers of the posterior thalamic peduncle. These occipitopontine fibers intermingled with the optic radiation and with the posterior thalamic peduncle fibers, at the level of the Meyer's loop, and then, continued their course in the lateral portion of the crus cerebri of the midbrain to reach the pontine nuclei. All these projection fibers reached their occipital cortical terminations by becoming part of the sagittal stratum.

Two patterns of angulation of the Meyer's loop were found in our study (Figure 4). The projection fibers in the 




Fig. 3. 3D DTT reconstruction of Meyer's loop (pink) in axial and left sagittal view. The 3D reconstructed fibers have been superimposed on axial and sagittal T1-weighted 3D turbo field echo (3D T1-TFE) images to enhance their precise anatomical localization and relationship with other anatomical structures. Fibers originating in the region of the lateral geniculate body, including pulvinar and the lateral aspect of crus cerebri of the midbrain, extended in an anterolateral direction into the anterior temporal region, before these fibers looped posteriorly and reached the occipital cortical region (left). All the fibers composing the Meyer's loop continued their course in the roof of the temporal horn, atrium and occipital horn of the lateral ventricle, and then these fibers turned medially, posterior to the occipital horn of the lateral ventricle, to reach the occipital cortex (right).

Meyer's loop extended in an anterolateral direction, from the region of the lateral geniculate bodies, to reach the anterior temporal region, where they looped sharply posteriorly, and coursed in the roof of the temporal and occipital horns of the lateral ventricles to reach the occipital regions, and thus formed a narrow angle Meyer's loop. This pattern of angulation of the Meyer's loop was demonstrated in 7 of our subjects. In 3 of our subjects, Meyer's loop was composed of projection fibers that extended laterally from the lateral geniculate bodies and displayed a more direct course in the anterior temporal region, before these fibers pursued a large loop, in the roof of the temporal horn of the lateral ventricles, and then continued their course in the roof of the occipital horn of the lateral ventricles to reach the occipital regions, and thus displayed a large angle Meyer's loop. Both raters showed the second pattern of

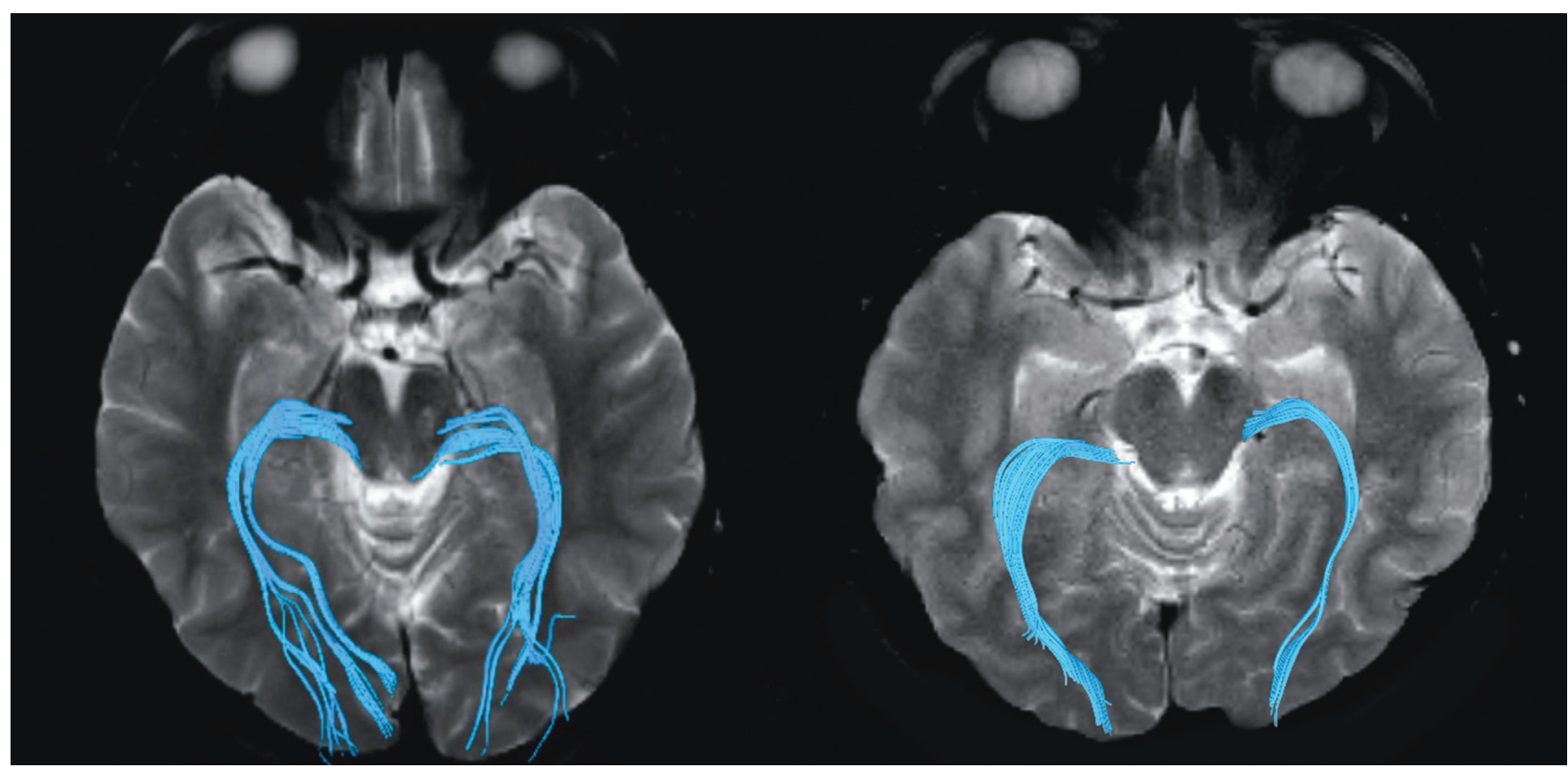

Fig. 4. 3D DTT reconstruction of Meyer's loop (light blue) in 2 different individuals to show two patterns of angulation. The 3D reconstructed fibers have been superimposed on axial T2-weighted turbo spin echo (T2-TSE SENSE) images. The projection fibers in the Meyer's loop extended in an anterolateral direction before looping sharply posteriorly to reach the occipital regions, and thus forming a narrow angle Meyer's loop (left). Meyer's loop was composed of projection fibers that extended laterally before pursuing a large loop to reach the occipital regions, thus displaying a large angle Meyer's loop (right). 
the Meyer's loop, a large angle Meyer's loop, with a similar morphology. The similar morphology of the reconstructed fibers participating in the formation of the Meyer's loop demonstrates reproducibility across observers.

Meyer's loop and related fibers in the anterior temporal region

An intricate relationship of the Meyer's loop with the uncinate fasciculus, occipitofrontal fasciculus, the anterior commissure and the inferior longitudinal fasciculus was demonstrated. The various projection fibers of the Meyer's loop intermingled and merged with both association and commissural fiber systems (Figure 5). The uncinate fasciculus is an association fiber system that connects orbital frontal regions with anterior temporal regions that was located anterior to the Meyer's loop in the anterior temporal region. The occipitofrontal fasciculus is an association fiber



\section{B}

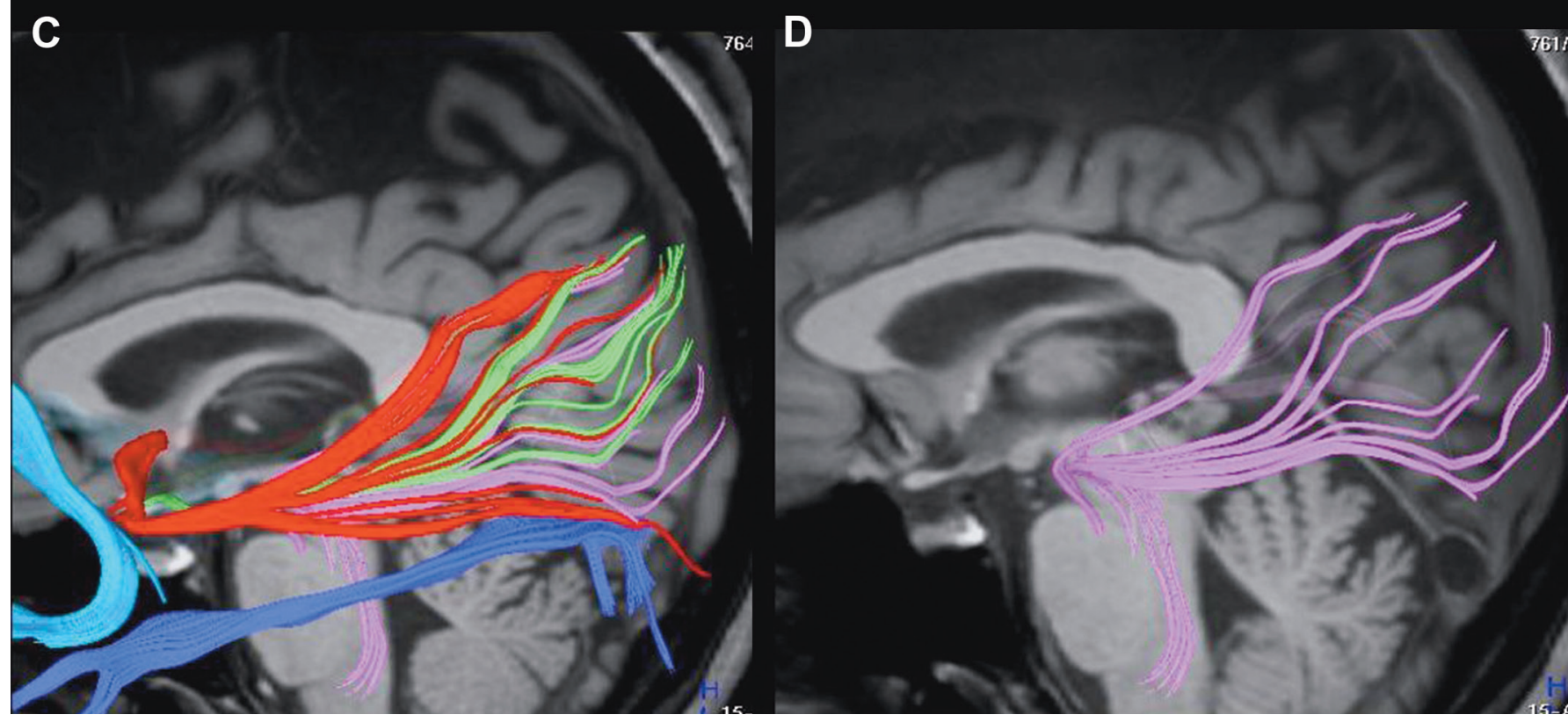

Fig. 5. 3D DTT reconstruction of the complex white matter fiber system related to Meyer's loop. The 3D reconstructed fibers have been superimposed on T1-weighted 3D turbo field echo (3D T1-TFE) images. A: Axial view of the 3D reconstructed white matter fibers that intermingle in the region of the Meyer's loop: posterior thalamic peduncle (pink) that includes optic radiation (green), occipitopontine fibers (pink), uncinate fasciculus (light blue), occipitofrontal fasciculus (yellow), the anterior commissure (red) and the inferior longitudinal fasciculus (blue). The optic radiation fibers (green) are in continuation of the optic tract (green). B: Sagittal view demonstrating the white matter fibers related to Meyer's loop in the anterior temporal region: posterior thalamic peduncle (pink) that includes optic radiation (green), uncinate fasciculus (light blue), occipitofrontal fasciculus (yellow), the anterior commissure (red) and the inferior longitudinal fasciculus (blue). Occipitofrontal fasciculus together with the lateral extension of the anterior commissure intermingle with the projection fibers in the Meyer's loop to form the sagittal stratum, and reach the occipital cortex. The inferior longitudinal fasciculus joins the inferior border of the sagittal stratum. C: Demonstration of the lateral extension of the anterior commissure fibers intermingled with the Meyer's loop in the anterior temporal region to form the sagittal stratum, on a sagittal view. D: Various projection fiber composing the Meyer's loop (pink) in a sagittal view: occipitopontine fibers, posterior thalamic peduncle and optic radiation. 
system that connects frontal and occipital regions. In the anterior temporal region, these fibers coursed laterally to the Meyer's loop. The anterior commissure presents a voluminous lateral extension that interconnects homologues temporal and occipital regions and a small anterior extension for homologues olfactory regions. The lateral extension of the anterior commissure coursed medially to the occipitofrontal fasciculus in the anterior temporal region, and then its fibers intercrossed and intermingled with the Meyer's loop to become a part of the sagittal stratum. The fibers of the occipitofrontal fasciculus, together with the lateral extension of the anterior commissure and the Meyer's loop projection fibers intermingled and merged in the compact layer of the sagittal stratum to reach their respective occipital cortical terminations. The inferior longitudinal fasciculus is an association fiber system that connects temporal and occipital regions that joined the inferior border of the sagittal stratum.

\section{Discussion}

This study applied a novel DTT technique to demonstrate the anatomy of the Meyer's loop in the anterior temporal region. After analysis and comparison of the methods and results of previous DTT studies, we hypothesized that our technique should be more accurate that using ROIs directly in the lateral geniculate body and calcarine cortex to reconstruct the Meyer's loop. This hypothesis requires validation in the future by a statistical comparison between different algorithms to verify the superiority of our technique. By using large ROIs in the region of the lateral geniculate body, including pulvinar of the thalamus and crus cerebri of the midbrain, and the occipital white matter the proposed method might allow for a more exhaustive demonstration of the complex projection fibers involved in the formation of the Meyer's loop. By using this technique, we were able to demonstrate that several projection fibers system, including optic radiation, posterior thalamic peduncle and occipitopontine fibers participate in the formation of the Meyer's loop and 2 patterns of the angulation of the Meyer's loop. With superimposition of the 3D reconstructed fibers on 3-planar T1-weighted 3D turbo field echo (3D T1-TFE) images, the composition, course and relations of the Meyer's loop with neighboring fibers could be revealed and evaluated in the $3 \mathrm{D}$ space, which could be useful for neurosurgical planning and intraoperative guidance.

Various projection fibers compose the Meyer's loop and not only the optic radiation

Recent fiber microdissection studies [2, 3] suggest that in the region of the lateral geniculate body there is a complex network of projection fibers for the temporal and occipital regions that participate in the formation Meyer's loop, contrary to the classical knowledge that Meyer's loop is composed exclusively of optic radiation. These authors conclude that all these various projection fibers form the Meyer's loop, and the optic radiation is only one of these several components. Moreover, individual projection fibers, such as the optic radiation, cannot be differentiated in the Meyer's loop, from the dense and complex network of projection fibers by using the fiber dissection technique.

To the authors knowledge, this is the first DTT study that evaluates the composition of the Meyer's loop comprehensively, by considering several projection fibers that pass in the confined region that includes the lateral geniculate body, pulvinar of thalamus and crus cerebri of midbrain to connect with occipital cortical regions, and may participate in the formation of the Meyer's loop. From our results, we hypothesized that various projection fibers, including the optic radiation, the posterior thalamic peduncle and the occipitopontine fibers may compose the Meyer's loop.

Many investigators used DTT to reconstruct and identify the course of the optic radiation by manually choosing two ROIs in the lateral geniculate body and the calcarine cortex respectively, technique that reconstructs exclusively the optic radiation fibers and does not consider the complex network or neighboring projection fibers in the region of the lateral geniculate body [11, 21, 24, 27]. The Meyer's loop reconstructed in these studies might be an overestimation of the actual structure, because of the increase probability of including the dense network of projection fibers intermingled with the optic radiation in the region of the lateral geniculate body. One recent DTT study specifically describes these complex relations between the projection fibers for the occipital region, among which the optic radiation is only one of the several component [18]. Although these authors, reconstructed and demonstrated the occipitopontine fibers together with the optic radiation, their technique was designed to specifically reconstruct individual optic radiation fibers and the occipitopontine fibers are considered as neighboring fibers rather that components of the Meyer's loop.

Two patterns of angulation of the Meyer's loop may exist in the human population

From our results we hypothesized, that 2 patterns of angulation of the Meyer's loop may exist in healthy subjects. The first pattern demonstrated in our study, a narrow angle Meyer's loop, was similar to the classical course. The second pattern, a large angle Meyer's loop, has not been previously described to authors knowledge. The hypothesis of a variable course of the Meyer's loop in the anterior temporal region has been discussed by a previous DTT study that demonstrated individual optic radiation fibers [18]. The authors of that study found that the optic radiation may present 2 patterns: one pattern in which the optic radiation participates in the formation of the classical Meyer's loop, and a second pattern where the optic radiation fibers presented a direct course to the occipital visual cortex without looping. The authors of this study concluded that although their results showed high reproducibility, the real anatomy of the Meyer's loop remains unclear, because of the technical limitations and algorithmic errors of the DTT, and should be investigated in future research with 
DTT in more subjects and in combination with cadaveric fiber dissection studies. The fact that optic radiation participates in the formation of the Meyer's loop or that it might be an exclusive component of the Meyer's loop has not been unequivocally proven and much controversy surrounds both the location and precise course of the optic radiation. From this perspective, a multicomponent structure of the Meyer's loop that includes various projection fibers supports the concept of a consistent existence of a Meyer's loop in the anterior temporal region, with a variable course, but with an unclear location of the optic radiation fibers among the various components. Although our results were consistent and reproducible, because of the intrinsic limitations of both cadaveric fiber dissection studies and DTT techniques, the anatomy of the Meyer's loop remains incomplete and controversial.

Limitations of the technique and future research regarding the anatomy of the Meyer's loop

DTT offers the only technique to study the anatomy of individuals, in vivo and noninvasively, but there are limitations of this technique that preclude an accurate appreciation of the ultimate anatomy of complex white matter structures such as the Meyer's loop. Demonstrating the anatomy of the Meyer's loop by DTT remains challenging because tracking algorithms and intrinsic technique limitations may fail to accurately reconstruct crossing or parallel fibers and fibers with a sharp curvature [11-16]. The results of the DTT might represent overestimations or underestimation of the actual structures, because tracts could be lost or mistakenly reconstructed by mathematical error of the algorithm, particularly the complex network of the fibers in the region of the lateral geniculate body or the Meyer's loop. Furthermore, DTT cannot provide information on a cellular-level axonal-connectivity. At the cellular level, the axons from individual neurons might merge or bifurcate, and their course might include multiple intercrossing between neighboring fibers systems, all happening within a voxel. Within a voxel, cellular level axonal information from multiple fiber systems is averaged and this leads to a significant amount of anatomical data loss. Future, more advanced, high-definition DTI technology that would enable a decrease in the voxel size down to the cellular-level connectivity, and improvements in the tracking algorithms might be able to clarify the anatomy of the Meyer's loop and asses its exact course and composition.

The ultimate anatomy of Meyer's loop requires future studies by using a combination of cadaveric white matter fiber dissection, histological-tracing studies applicable in humans and DTT. White matter fiber dissection is a practical technique to demonstrate the Meyer's loop and related fibers, with the advantage of obtaining anatomical information that is implicitly accepted as true. It is considered the gold standard for validation of the anatomical details of the white matter. However, both white matter fiber dissection and currently available histologic techniques applicable in humans have limitations, particularly when dealing with complex fiber systems, such as the Meyer's loop. Our observations from DTT, are based on a population of 10 healthy subjects, and future work, on larger populations, and by comparing different deterministic and probabilistic algorithms are required to validate our hypothesis. Patients with pathological conditions are different from healthy subjects. Future work, by using DTT to study the anatomical details of the Meyer's loop in different kinds of pathologies involving the temporal lobe and to compare the differences between healthy subjects and patients may have potential practical applications in preoperative planning for neurosurgical procedures and intraoperative guidance.

\section{Conclusions}

DTT provides a complimentary method to study the details of the anatomy of the Meyer's loop in vivo. A complex network of white matter fibers that includes several projection fibers system, the optic radiation, posterior thalamic peduncle and occipitopontine fibers, participate in the formation of the Meyer's loop and 2 patterns of the angulation of the Meyer's loop may exist in healthy subjects. Meyer's loop is a complex anatomical structure with anatomic details that remain obscure and future validation of our anatomical findings is required. Future work by using more advanced, high-definition DTT might be able to produce more comprehensive and accurate demonstrations of the intricate white matter architecture, particularly for highly complex structures such as the Meyer's loop, that can be applied in planning and guidance for neurosurgical procedures.

\section{Acknowledgements}

This paper was partly supported by the Sectorial Operational Program Human Resources Development (SOP HRD), financed from the European Social Fund and by the Romanian Government under the contract number POSDRU 80641.

\section{References}

1. Meyer A. The connections of the occipital lobes and the present status of the cerebral visual affections. Trans Assoc Am Physicians. 1907;22:7-16.

2. Türe $U$, Yașargil MG, Friedman $A H$, Mefty $A I O$. Fiber dissection technique: lateral aspect of the brain. Neurosurgery. 2000;47:417-426.

3. Yașargil MG, Türe $U$, Yasargil DC. Impact of temporal lobe surgery. J Neurosurg. 2004;101:725-738.

4. Basser PJ, Mattiello J, LeBihan D. MR diffusion tensor spectroscopy and imaging. Biophys J. 1994; 66:259-267.

5. Basser PJ, Mattiello J, LeBihan D. Estimation of the effective self-diffusion tensor from the NMR spin echo. J Magn Reson B. 1994;103:247-254.

6. Basser PJ, Pajevic S, Pierpaoli C, et al. In vivo fiber tractography using DT-MRI data. Magn Reson Med. 2000; 44:625-632.

7. Mori S, Zhang J. Principles of diffusion tensor imaging and its applications to basic neuroscience research. Neuron. 2006; 51:527-539.

8. Conturo TE, Lori NF, Cull TS, et al. Tracking neuronal fiber pathways in the living human brain. Proc Natl Acad Sci USA. 1999; 96:10422-10427.

9. Jones DK. Studying connections in the living human brain with diffusion MRI. Cortex. 2008;44:936-952.

10. Mori S, van Zijl PC. Fiber tracking: principles and strategies - a technical review. NMR Biomed. 2002;15:468-480.

11. Yamamoto T, Yamada K, Nishimura T, Kinoshita S. Tractography to depict three layers of visual field trajectories to the calcarine gyri. ANJR Am J Neuroradiol. 2005;140:781-785. 
12. Okada T, Miki Y, Kikuta K, et al. Diffusion tensor fiber tractography for arteriovenous: quantitative analyses to evaluate the corticospinal tract. ANJR Am J Neuroradiol. 2007; 28:1107-1113.

13. Ciccarelli O, Toosy AT, Hickman SJ, al E. Optic radiation changes after optic neuritis detected by tractography-based group mapping. Hum Brain Mapp. 2005;25:308-316.

14. Kikuta K, Takagi Y, Nozaki K. Early experience with 3-T magnetic resonance tractography in the surgery of cerebral arteriovenous malformations in and around the visual pathways. Neurosurgery. 2006;45:331-337.

15. Powell HW, Parker GJ, Alexander DC, et al. MR tractography predicts visual field defects following temporal lobe resection. Neurology. 2005;34:596-599.

16. Taoka K, Sakamoto M, Iwasaki S. Diffusion tensor imaging in cases of visual field defect after anterior temporal lobectomy. AJNR Am J Neuroradiol. 2005:67:797-803.

17. Kier EL, Staib LH, Davis LM, Bronen RA. MR imaging of the temporal stem: anatomic dissection tractography of the uncinate fasciculus, inferior occipitofrontal fasciculus, and Meyer's loop of the optic radiation. AJNR Am J Neuroradiol. 2004; 25:677-691.

18. Wu W, Rigolo L, O'Donnell LJ, et al. Visual pathway study using in vivo diffusion tensor imaging tractography to complement classic anatomy. Neurosurgery. 2012;70:ons145-ons156.

19. Mori S, Kaufmann WE, Davatzikos C, et al. (2002) Imaging cortical association tracts in human brain. Magn Reson Imag 47:215-223.
20. Kovanlikaya I, Firat Z, Kovanlikaya A, et al. Assessment of the corticospina tract alterations before and after resection of brainstem lesions using diffusion tensor imaging (DTI) and tractography at 3T. Eur J Radiol. 2005;77:383-391.

21. Nilsson D, Starck G, Ljungberg M, et al. Intersubject variability in the anterior extent of the optic radiation assessed by tractography. Epilepsy Research. 2007;77:11-16.

22. Nieuwenhuys R, Voogd J, van Huijzen C. The Human Central Nervous System, 4 ed. Springer-Verlag, Berlin, 2008.

23. Saeki N, Fujimoto N, Kubota M, Yamaura A. MR demonstration of partial lesions of the lateral geniculate body and its functional intra-nuclear topography. Clin Neurol Neurosurg. 2003; 106:28-32.

24. Sherbondy AJ, Dougherty RF, Napel S, Wandell BA. Identifying the human optic radiation using diffusion tensor imaging and fiber tractography. J Vis. 2008;8:12.1-12.11.

25. Chen X, Weigel D, Ganslandt O, et al. Prediction of visual field deficits by diffusion imaging in temporal lobe epilepsy surgery. Neurolmage. 2009;45:286-297.

26. Barton JJS, Hefter R, Chang B, et al. The field defects of anterior temporal lobectomy: a quantitative reassessment of Meyer's loop. Brain. 2005;128:2123-2133

27. Lilja Y, Ljungberg M, Starck G, et al. Visualizing Meyer's loop: A comparison of deterministic and probabilistic tractography. Epilepsy Research. 2014;108:481-490 